These results, say the authors, are further evidence for a pathogenetic role of EPC dysregulation in diabetic vasculopathy. They believe that therapy against diabetic atherosclerotic complications should include methods for increasing the number of EPCs and improving their function. Furthermore, $\mathrm{CD} 4^{+} \mathrm{KDR}^{+} \mathrm{EPC}$ count could be used as a novel biomarker of peripheral atherosclerosis in patients with diabetes.

Original article Fadini GP et al. (2006) Number and function of endothelial progenitor cells as a marker of severity for diabetic vasculopathy. Arterioscler Thromb Vasc Biol 26: 2140-2146

\section{Facilitation of coronary sinus cannulation using fiberoptic endocardial visualization}

The success rate of cardiac resynchronization therapy (CRT) implantation, according to a recent study, could be improved by the use of an endocardial visualization catheter (EVC), which allows direct, real-time visualization of intracardiac structure.

Despite improvements in the techniques used, a significant number of CRT implantations fail, mainly because of the presence of complex anatomy that limits the ability to cannulate the coronary sinus (CS). In this study, 58 consecutive patients (mean age $72 \pm 12$ years; ejection fraction $26.2 \pm 7.0 \%$; NYHA class 2.9) requiring CRT implantation for a range of standard indications were imaged during the procedure using a steerable fiberoptic EVC. Visualization of the CS ostium was successful in all cases and the CS was cannulated in 57 (98.3\%) patients. The mean time from EVC insertion to $C S$ visualization was $6 \pm 5 \mathrm{~min}$, and the mean total time to CS access was $8 \pm 6 \mathrm{~min}$. Left ventricle implantation was accomplished in 55/58 (94.8\%) patients, including 3 patients who had a previous history of failed left ventricular lead implantation. The three unsuccessful implantations in this study were caused by dissection of the CS during lead delivery in one patient, prevention of lead sheath placement because of tortuous angulations in a second, and the presence of a valve in the third.

The authors suggest that fiberoptic imaging using this technique can aid CS access and left ventricular lead placement by allowing visualization of right atrial anatomy during CRT implantation.
Original article Anh DJ et al. (2006) Early human experience with use of a deflectable fiberoptic endocardial visualization catheter to facilitate coronary sinus cannulation. Heart Rhythm 3: 875-878

\section{Tissue Doppler-derived E/E' predicts clinical outcome in nonvalvular atrial fibrillation}

The ratio of early transmitral flow velocity $(E)$ to early diastolic mitral annular velocity $\left(E^{\prime}\right)$ is a novel index of left ventricular filling pressure and has been shown to predict prognosis in patients with a variety of heart diseases.

To investigate the usefulness of the Dopplerderived index of left ventricular filling pressure, $E / E^{\prime}$, in predicting the clinical outcome of patients with nonvalvular atrial fibrillation, Okura and colleagues divided 230 consecutive patients into 2 groups based on their $E / E^{\prime}$ value. Group A comprised patients with $E / E^{\prime} \leq 15$ $(n=140)$ and group B comprised patients with $E / E^{\prime}>15(n=90)$. After an average follow-up of 245 days, $21(9.1 \%)$ patients had died, of whom significantly more were in group $B$ than group A (15 [16.7\%] versus 6 [4.3\%] patients; $P=0.0015)$. Both cardiac death and congestive heart failure were more common in group B than in group A (10 [11.1\%] versus 2 [1.4\%] patients, and 16 [17.8\%] versus 8 [5.7\%] patients; $P<0.01$ for both). Cumulative survival was also significantly lower in group $B$ than in group $A$ (log rank $P=0.0013)$. Multivariate logistic regression analysis demonstrated that only E/E' (odds ratio 1.05, 95\% Cl 1.01-1.11; $P=0.03$ ) and age (odds ratio 1.06, 95\% Cl 1.01$1.11 ; P=0.02$ ) were independent predictors of mortality.

On the basis of these findings, the authors conclude that Doppler-derived $E / E^{\prime}$ is a useful predictor of clinical outcome in patients with nonvalvular atrial fibrillation.

Original article Okura H et al. (2006) Tissue Dopplerderived index of left ventricular filling pressure, E/E', predicts survival of patients with non-valvular atrial fibrillation. Heart 92: 1248-1252

\section{ALIAS trial of high-dose human albumin for acute stroke produces encouraging results}

Promising results were recently reported for the ALIAS (Albumin in Acute Stroke) Pilot Trial-a phase I, open-label, dose-escalation 